\title{
Interactive comment on "Implementing a sectional scheme for early aerosol growth from new particle formation in the Norwegian Earth System Model v2: comparison to observations and climate impacts" by Sara M. Blichner et al.
}

Anonymous Referee \#2

Received and published: 18 December 2020

The manuscript "Implementing a sectional scheme for early aerosol growth from new particle formation in the Norwegian Earth System Model v2: comparison to observations and climate impacts" introduces a new method for calculating the formation and growth of newly formed particles. The method applies the sectional approach for describing the smallest particles together with the modal method for the larger particles. The paper is well and clearly written and is definitely in the scope of Geoscientific Model Development. The method presented here is definitely relevant for the global aerosol modelling community and it can be used in any modal aerosol model. I can 
recommend publishing the manuscript after the following issues are addressed:

- The confidence in the improved model could be strengthened by demonstrating its performance in a 0-dimensional framework comparing it to for example a high resolution sectional model.

- The differences between different model version are discussed from the view point of aerosol microphysics. However, for example in-cloud impact scavenging affects strongly the number of small particles. I am not familiar with the size dependence in CAM-Nor wet deposition scheme; can wet scavenging cause differences between the simulated aerosol concentrations in different model versions?

- On Page 17, Line 510 onward, the explanation between the different behavior is slightly ambiguous, especially the last sentence of the paragraph: "Additionally, in highly polluted areas, an increased number of particles may inhibit activation because more particles compete for the same water vapor". Does it want to say that in polluted areas, newly formed particles decrease supersaturation so much that it suppresses activation? If so, my understanding that in order to suppress the number of activated particles by increasing the number of particles, number concentrations have to be extremely high and would occur in very few parts of the atmosphere.

Does also the modal representation affect this behavior? With a high number of NPF particles entering the smallest mode, the mode will shift to smaller sizes artificially suppressing activation.

\section{Technical comments:}

- Page 2, Line 49: Correct "depend" to "dependent"

- The model is referred to as CAM6-Nor and CAM-Nor6. On Page 9, Line 256, CAM6-Oslo is mentioned. Please correct the wrong namings. 
Interactive comment on Geosci. Model Dev. Discuss., https://doi.org/10.5194/gmd-2020$357,2020$.
GMDD

Interactive

comment 